\title{
Positive Controllability of Large-Scale Networks
}

\author{
Gustav Lindmark and Claudio Altafini
}

Conference Publication

\section{Tweet}

N.B.: When citing this work, cite the original article.

Original Publication:

Gustav Lindmark and Claudio Altafini, Positive Controllability of Large-Scale Networks, Proceedings of the 2016 European Control Conference, 2016, pp.819-824.

Copyright:

\section{http://ieee.org}

Postprint available at: Linköping University Electronic Press

http://urn.kb.se/resolve?urn=urn:nbn:se:liu:diva-130780

10U UNKKÖPNGS 


\title{
Positive Controllability of Large-Scale Networks
}

\author{
Gustav Lindmark and Claudio Altafini
}

\begin{abstract}
In this paper, we study the problem of controlling large scale networks with controls which can assume only positive values. Given an adjacency matrix $A$, an algorithm is developed that constructs an input matrix $B$ with a minimal number of columns such that the resulting system $(A, B)$ is positively controllable. The algorithm combines the graphical methods used for structural controllability analysis with the theory of positive linear dependence. The number of control inputs guaranteeing positive controllability is near optimal.
\end{abstract}

\section{INTRODUCTION}

The concept of controllability has played a fundamental role in system theory since it was first introduced by Kalman in [1]. A recent trend in the literature is to investigate the controllability of large-scale complex networks such as those appearing in a broad spectrum of scientific disciplines, ranging from Biology to Social Sciences, from Technology to Engineering. When no a-priori information is available, then an interesting problem investigated for instance in [2], [3] is to determine what is the minimal number of driver nodes that allows to guarantee controllability.

When in the graph that represents the network only the location of the edges connecting the nodes is known but limited information is available on the edge weights, then one can resort for example to the notion of structural controllability [4], [5]. The main idea behind structural controllability is that, assuming a linear dynamics, controllability of such "graphical" systems is a generic property that holds for almost all values of the parameters (i..e. edge weights). In [2] this idea is applied to various networks, and asymptotic expressions for the number of driver nodes for different network types are derived. When instead a set of weights is given, then the construction of a minimal set of driver nodes can rely upon other well-established controllability tests. For instance in [6] a method based on the PBH test is introduced, while in [3] a thorough estimate of the minimal number of driver nodes is computed. Other characterizations of such "input selection" problem appear in [7], [8], [9].

In many of the different fields in which controllability of large networks can be investigated, even assuming that each node can be treated as a driver node, the control action that the driver nodes can exert on the system is not free but intrinsically constrained. The most common form of constraint is that the input is unidirectional, i.e., can assume only positive or negative values but not both. For instance, in a biological network, nodes correspond to molecular components, and a

G. Lindmark and C. Altafini are with the Division of Automatic Control, Dept. of Electrical Engineering, Linköping University, SE58183, Linköping, Sweden. email: gustav.lindmark@liu.se, claudio.altafinieliu.se drug acting on a molecule can be considered a control input. However, its mode of action is to either activate its target or to inhibit it, not both. Complex networks in which inputs are naturally unidirectional occur in many other domains, such as for instance in flow networks in transportation or in power networks. Taking for example a power network, loads are absorbing power but not producing it, while generators normally play the opposite role. Hence both are naturally associated to unidirectional control actions if the purpose is to control the power flow over the network. In all of these examples the input set is constrained, and in particular unidirectional. The notion of positive controllability, which capture the idea of controlling systems only though positive controls, is the one that can be used in this context. It also has a long history, see Chapter 5 of [10] for a survey. General results on controllability with constrained inputs were formulated by Brammer in [11]. Brammer condition requires to specify which eigenvectors of the state update matrix $A$ are real. As such, it is not a generic condition in the sense of structural controllability, because it requires the knowledge of the numerical value of the entries of $A$. See [12], [13] for other related material.

The aim of this paper is to translate the positive controllability conditions of [11] into an efficient computational method for the calculation of a minimal number of control inputs achieving positive controllability of a given matrix $A$ representing a large-scale network. The algorithms we propose combine the graph-based properties of structural controllability with the theory of positive linear dependence [14], and can be applied to any complex network in which the control inputs act either positively or negatively on a node.

A computational study of the results of our algorithm is carried out for random Erdős-Rényi networks with random edge weights. It is found that variations in the numerical values of the system parameters lead to small differences in the number of required control inputs. Furthermore, the number of control inputs required are tightly related to certain network properties.

The rest of the paper is organized as follows: In Section II, definitions are given and some results on structural controllability as well as controllability with restrained control inputs are revised. In Section III, conditions for positive controllability are reformulated in more suitable terms than in [11]. Section IV presents the main contribution of this paper, an algorithm that constructs the control input matrix $B$ with a near-minimal number of inputs. In Section $\mathrm{V}$ the algorithm is applied to random networks, and the results are related to network properties. 


\section{BACKGROUND AND BASIC DEFINITIONS}

\section{A. Notation}

Given a matrix $M$, let $M[k]$ denote the $k$-th column of $M$. When used on a transposed matrix, $M^{T}[k]$ shall be interpreted as $\left(M^{T}\right)[k]$. Given a set of indices $\mathcal{P}=\left\{p_{1}, \ldots, p_{m}\right\}$, $M[\mathcal{P}]$ denotes the matrix $\left[M\left[p_{1}\right] \quad \ldots \quad M\left[p_{m}\right]\right] . M[i, j]$ denotes the element on row $i$ and column $j$ of the matrix $M$. Given the two vectors $v_{1}$ and $v_{2},\left\langle v_{1}, v_{2}\right\rangle$ is their Euclidean product.

A (directed) graph $\mathcal{G}$ is indicated by the pair of its nodes and edges, $\mathcal{U}$ and $\mathcal{E}$, or, if it is necessary to specify the edge weights, by the adjacency matrix $A$, i.e., $\mathcal{G}=\mathcal{G}(A)$. A strongly connected component of $\mathcal{G}$ that has no incoming edge from any other strongly connected component will be called a Rooted Connected Component (RCC).

\section{B. Controllability}

Consider the system

$$
\begin{aligned}
S: \dot{x} & =A x+B u, \\
u & \in \Omega,
\end{aligned}
$$

with $A \in \mathbb{R}^{n \times n}$ and $B \in \mathbb{R}^{n \times m}$. The admissible controls are all vector functions $u(t)$ taking value in the control restraint set, $\Omega \subset \mathbb{R}^{m}$. The system (1) is controllable if for each pair of $x_{0}$ and $x_{1}$ in $\mathbb{R}^{n}$, there exist an admissible control, $u(t) \in \Omega$, defined on some finite interval $0 \leq t \leq t_{1}$, which steers $x_{0}$ to $x_{1}$. The reachable set $\mathcal{R}(t)$ is the set of all points in $\mathbb{R}^{n}$ to which the origin can be steered at time $t$ by an admissible control $u \in \Omega$. The reachable set $\mathcal{R}_{\infty}$ is the union of $\mathcal{R}(t)$ over all positive $t$. The system is nullcontrollable if there exists an open set $\Gamma$ in $\mathbb{R}^{n}$ containing the origin for which any $x_{0} \in \Gamma$ can be controlled to $x_{1}=0$ in finite time. Let $C(A, B)$ denote the controllability matrix $\left[B A B \ldots A^{n-1} B\right]$.

In this paper, a system with only positive controls is studied,

$$
\begin{aligned}
S_{r}: \dot{x} & =A x+B u, \\
u & \geq 0,
\end{aligned}
$$

i.e., case the control restraint set is $\Omega=\mathbb{R}_{+}^{m}$.

\section{Structural Controllability}

Here we present a brief summary of some basic results on structural controllability. See [7], [4] for more details on this topic. A linear time-invariant system with parametrized entries

$$
S_{\Delta}: \dot{x}=A_{\Delta} x+B_{\Delta} u,
$$

is said structured if the entries of $A_{\Delta}$ and $B_{\Delta}$ are either fixed zeros or independent parameters (not related by algebraic equations). Let $\Delta=\left\{\delta_{1}, \delta_{2}, \ldots, \delta_{k}\right\}$ denote such independent parameters. A graph $\mathcal{G}_{\Delta}=(\mathcal{U}, \mathcal{E})$ can be associated with $S_{\Delta}$. In $\mathcal{G}_{\Delta}$, each state and input is represented with a node,

$$
\mathcal{U}=\left\{u_{1}, \ldots, u_{m}, x_{1}, \ldots, x_{n}\right\}
$$

while the nonzero elements in $A_{\Delta}$ and $B_{\Delta}$ yield the edges of $\mathcal{G}_{\Delta}$

$$
\mathcal{E}=\left\{\left(x_{j}, x_{i}\right) \mid A_{\Delta}[i, j] \neq 0\right\} \cup\left\{\left(u_{p}, x_{i}\right) \mid B_{\Delta}[i, p] \neq 0\right\} .
$$

Controllability is a generic property of a structured system, i.e. it is true for almost all values of the parameters [15]. Such a notion is called structural controllability. The generic rank of the structured matrix $\left[\begin{array}{ll}A_{\Delta} & B_{\Delta}\end{array}\right]$ is defined to be the maximum rank that $\left[\begin{array}{ll}A_{\Delta} & B_{\Delta}\end{array}\right]$ can attain as a function of all the free parameters in $\Delta$.

Theorem 1 ([4]). The system $S_{\Delta}$ is structurally controllable if and only if

i) the graph of the system, $\mathcal{G}_{\Delta}$, is input connected,

ii) the generic rank of $\left[\begin{array}{ll}A_{\Delta} & B_{\Delta}\end{array}\right]=n$.

In the following, conditions i) and ii) of Theorem 1 will be referred to as the input connected condition and the rank condition. The input connected condition is met when all RCC's of the graph $\mathcal{G}_{\Delta}$ are single nodes which are inputs. The generic rank, $d$, of $\left[A_{\Delta} B_{\Delta}\right]$ can be computed using the Dulmage-Mendelsohn (DM) decomposition as in [7]. By doing that, a number of results follow:

i) The generic rank is obtained, and thereby also the rank deficiency, $d_{r}=n-d$.

ii) It is possible to identify states that must be controlled (by adding additional inputs acting on them).

iii) It is possible to identify states such that $d_{r}$ is reduced by adding a control to them.

\section{Positive Linear Dependence}

The theory on positive linear dependence provides a useful framework for our controllability analysis with positive inputs. Some important results from [14] are recalled here. A positive combination of vectors $a_{1}, \ldots, a_{r}$ is a linear combination $\eta_{1} a_{1}+\cdots+\eta_{r} a_{r}$ with $\eta_{i} \geq 0$. An algebraic equation $\eta_{1} a_{1}+\cdots+\eta_{r} a_{r}=0$ with $\eta_{i} \geq 0$ is said a positive relation, and with $\eta_{i}>0$ it is called a strictly positive relation.

A convex cone $\mathcal{C}$ is the set of all positive combinations of a finite set of vectors, not all zero

$$
\mathcal{C}=\left\{\sum_{i=1}^{r} \eta_{i} a_{i} \mid \eta_{i} \in \mathbb{R}^{+}\right\} .
$$

The set of vectors $a_{1}, \ldots, a_{r}$ is said to positively span the convex cone $\mathcal{C}$. For a matrix $M \in \mathbb{R}^{p \times q}, \mathcal{C}(M) \subseteq \mathbb{R}^{p}$ denotes the convex cone of positive combinations of its columns.

The vectors $a_{1}, \ldots, a_{r} \in \mathbb{R}^{n}$ positively span $\mathbb{R}^{n}$ if for any $x \in \mathbb{R}^{n}, \exists \eta_{i} \geq 0$ s.t. $x=\eta_{1} a_{1}+\cdots+\eta_{r} a_{r}$. To avoid confusion, linearly span is sometimes used for spanning in the usual sense. The following theorems from [14] will be useful:

Theorem 2 Let $\left\{a_{1}, \ldots, a_{r}\right\}$ linearly span $\mathbb{R}^{n}$. Then the following are equivalent: 
i) $\left\{a_{1}, \ldots, a_{r}\right\}$ positively span $\mathbb{R}^{n}$.

ii) There is some strictly positive relation between the $a_{i}$, $i=1, \ldots, r$.

To positively span $\mathbb{R}^{n}$, at least $n+1$ vectors are needed.

Theorem 3 The vectors $a_{1}, \ldots, a_{r}$ positively span $\mathbb{R}^{n}$ if and only if, for every non-zero $b \in \mathbb{R}^{n}$, there is an $i \in\{1, \ldots, r\}$ such that $\left\langle b, a_{i}\right\rangle>0$.

Theorem 3 implies that if $a_{1}, \ldots, a_{r}$ do not positively span $\mathbb{R}^{n}$, then there is a $b$ such that $\left\langle b, a_{i}\right\rangle \leq 0 \forall i \in\{1, \ldots, r\}$. Given a set of vectors $a_{1}, \ldots, a_{r} \in \mathbb{R}^{n}$, the problem of determining if they positively span $\mathbb{R}^{n}$ can be done by solving a linear optimization problem. [16]

\section{E. Positive Controllability}

Conditions for controllability of systems with constrained inputs are derived in [11] and further investigated in [12]. The results are not generic but specific to a pair $(A, B)$. The main theorem states that the following conditions are necessary and sufficient for null-controllability of (1):

Theorem 4 ([11]) Consider the system (1) satisfying the following conditions:

i) There exists $u \in \Omega$ satisfying $B u=0$.

ii) The convex hull of $\Omega$ has nonempty interior in $\mathbb{R}^{m}$.

Then (1) is null-controllable if and only if

iii) The matrix $C(A, B)$ has rank $n$.

iv) There is no real eigenvector $v$ of $A^{T}$ satisfying $\langle v, B u\rangle \leq 0$ for all $u \in \Omega$.

A real eigenvector denotes an eigenvector associated with a real eigenvalue, as in [11]. An alternative but equivalent formulation of $i v$ ) in Theorem 4 is:

$\left.i v^{\prime}\right)$ For any real eigenvector $v$ of $A^{T}$ there must be a $u \in \Omega$ such that $\langle v, B u\rangle>0$.

For the system $S_{r}, \Omega=\mathbb{R}_{+}^{m}$ and condition ii) is met, and, since $u=0$ is admissible, clearly also condition i) holds. Furthermore, for $S_{r}$, null-controllability implies controllability since the inputs can be arbitrarily scaled, enlarging the set $\Gamma$ to cover $\mathbb{R}^{n}$. We shall refer to this case as positive controllability.

\section{Reformulation of Positive Controllability}

Let $\lambda_{i}, i=1, \ldots, l$, be the distinct real eigenvalues of $A^{T} \in \mathbb{R}^{n \times n}$, and $\mu_{i}$ their geometric multiplicity. Denote $\left\{v_{i_{1}}, \ldots, v_{i_{\mu_{i}}}\right\}$ the linearly independent real eigenvectors that linearly span the eigenspace of $\lambda_{i}$. Assume that $B \in$ $\mathbb{R}^{n \times m}$ is given. Define

$$
V_{i}=\left[\begin{array}{llll}
v_{i_{1}} & v_{i_{2}} \ldots v_{i_{\mu_{i}}}
\end{array}\right],
$$

and

$$
\rho_{i}=V_{i}^{T} B \in \mathbb{R}^{\mu_{i} \times m}, \quad i=1, \ldots, l .
$$

Consider first the case when all real eigenvalues are simple, i.e., $\mu_{i}=1, i=1, \ldots, l$, and $V_{i}=v_{i_{1}}$. In this case we have $\rho_{i}=v_{i_{1}}^{T} B \in \mathbb{R}^{1 \times m}$.
Proposition 1 A necessary condition for positive controllability of the system $S_{r}$ is that each $\rho_{i}, i \in\left\{1, \ldots, l \mid \mu_{i}=1\right\}$ has both positive and negative entries. If $\mu_{i}=1 \forall i=1, \ldots l$, and $\operatorname{rank}(C(A, B))=n$, then this condition is also sufficient for positive controllability.

Proof: Introduce the scaled eigenvector $v_{i}=\alpha_{i} v_{i_{1}}$, $\alpha_{i} \in \mathbb{R}$. Condition $i v$ ) of Theorem 4 applied to $v_{i}$ requires that there is a $u \geq 0$ such that

$$
\begin{aligned}
& \left\langle v_{i}, B u\right\rangle>0, \\
& \Longleftrightarrow v_{i}^{T} B u=\alpha_{i} \rho_{i} u>0 .
\end{aligned}
$$

Start with assuming that the scalar $\alpha_{i}>0$. Since $u \geq 0$, equation (9) holds only if there is some positive entry in $\rho_{i}$. Infact if $\rho_{i} \leq 0$, then $\left\langle v_{i}, B u\right\rangle \leq 0$ for any admissible $u$. Assume that the $s$ th entry is $\rho_{i}[s]>0$. Selecting $u[s]>0$ and $u[j]=0 \forall j \neq s$ gives $\rho_{i} u>0$, i.e. $\left\langle v_{i}, B u\right\rangle>0$ and condition $i v$ ) is met for $v_{i}=\alpha_{i} v_{i_{1}}, \alpha_{i}>0$.

Similarily, if we assume $\alpha_{i}<0$, one comes to the conclusion that condition $i v$ ) is met for $v_{i}=\alpha_{i} v_{i_{1}}$ only if there is a negative entry in $\rho_{i}$.

Since any $v_{i}=\alpha_{i} v_{i_{1}}$ is an eigenvector $\forall \alpha_{i} \in \mathbb{R} \backslash\{0\}$, it is necessary that $\rho_{i}, i \in\left\{1, \ldots, l \mid \mu_{i}=1\right\}$ has both positive and negative entries.

If $\mu_{i}=1 \forall i=1, \ldots l$, then denote $\mathcal{V}=\left\{\alpha_{1} v_{1}, \ldots, \alpha_{l} v_{l}\right\}$ the set of all real eigenvectors of $A^{T}$. For each $\rho_{i}$ with both positive and negative entries we know that there is a $u \geq$ 0 such that $\left\langle\alpha_{i} v_{i_{1}}, B u\right\rangle>0$ holds. If this is the case $\forall i$, then for any $v \in \mathcal{V}$ there is a $u \geq 0$ such that $\langle v, B u\rangle>$ 0 , and condition iv) is met. With $\operatorname{rank}(C(A, B))=n$, the conditions of Theorem 4 are then satisfied and the system is controllable.

Now consider the case in which $\mu_{i}>1$, and $v_{i_{1}}, \ldots v_{i_{\mu_{i}}}$ linearly span the eigenspace of $\lambda_{i}$. From (7), any eigenvector $v_{i}$ associated with $\lambda_{i}$, i.e. $A^{T} v_{i}=\lambda_{i} v_{i}$, can be written as a linear combination

$$
v_{i}=V_{i} \alpha_{i}, \alpha_{i} \in \mathbb{R}^{\mu_{i}} .
$$

Notice that $\rho_{i}$ in (8) now is a $\mu_{i} \times m$ matrix with as many rows as the dimension of the eigenspace of $\lambda_{i}$ and as many columns as there are inputs. Condition iv) of Theorem 4 can now be reformulated in a way that is more practical for our purposes.

Theorem 5 For any real eigenvector $v$ of $A^{T}$ there is a $u \geq 0$ such that $\langle v, B u\rangle>0$, if and only if the columns of $\rho_{i}$ positively span $\mathbb{R}^{\mu_{i}} \forall i=1, \ldots, l$.

Proof: "If part". Denote $\mathcal{V}=\left\{V_{1} \alpha_{1}, \ldots, V_{l} \alpha_{l}\right\}, \alpha_{i} \in$ $\mathbb{R}^{\mu_{i}}, i \in\{1, \ldots, l\}$, the set of all real eigenvectors of $A^{T}$. Selecting any eigenvector $v \in \mathcal{V}$, from (10),

$$
\begin{aligned}
\langle v, B u\rangle & =\left\langle\alpha_{i}, \rho_{i} u\right\rangle \\
& =\sum_{j=1}^{m}\left\langle\alpha_{i}, \rho_{i}[j]\right\rangle u[j] .
\end{aligned}
$$


By assumption any point in $\mathbb{R}^{\mu_{i}}$ is a positive combination of the columns of $\rho_{i}$ since they positively $\operatorname{span} \mathbb{R}^{\mu_{i}}$. For example, given $\alpha_{i}$, there is a positive combination with $u \geq 0$ s.t. $\rho_{i} u=\alpha_{i}$. From (11a) we then have $\left.\langle v, B u\rangle=\left\langle\alpha_{i}, \alpha_{i}\right\rangle\right\rangle$ 0 .

"Only if part". By contradiction, assume the columns of $\rho_{i}$ do not positively span $\mathbb{R}^{\mu_{i}}$. We will show that there is a $v \in \mathcal{V}$ for which $\langle v, B u\rangle \leq 0, \forall u \geq 0$. Consider (11b) and use Theorem 3 with $\rho_{i}[j], j=1, \ldots, m$, instead of the vectors $a_{1}, \ldots, a_{r}$, and $\alpha_{i}$ instead of $b$. Since $\rho_{i}[j], j=$ $1, \ldots, m$, do not positively span $\mathbb{R}^{\mu_{i}}$, there is a $\alpha_{i}$ for which $\left\langle\alpha_{i}, \rho_{i}[j]\right\rangle \leq 0 \forall j$. Used in (11b), this gives $\langle v, B u\rangle \leq 0$ which is a contradiction.

Theorem 5 is a generalization of Proposition 1. The condition of Proposition 1 , namely that $\rho_{i} \in \mathbb{R}^{1 \times m}$ has both positive and negative entries, in fact means that the columns of $\rho_{i}$ positively span $\mathbb{R}$. The following Corollary summarizes the results of this Section.

\section{Corollary 1 The system (2) is positively controllable iff}

i) The columns of $\rho_{i}$ positively span $\mathbb{R}^{\mu_{i}} \forall i=1, \ldots, l$.

ii) The matrix $C(A, B)$ has rank $n$.

\section{A Construction Procedure for Control INPUTS}

Assume that the system matrix $A$ is given. We want to construct a matrix $B$ such that the system $(A, B)$ is positively controllable with as few control inputs as possible. In the construction of $B$, we assume that we are free to add control inputs that act on any single state either positively or negatively.

\section{A. Structure of the Input Matrix}

Let $b_{j}$ be the column vector for an input acting positively on state $x_{j}, b_{j}[j]=1$, and only on it: $b_{j}[i]=0 \forall i \neq j$. It is not a restriction to assume a value of 1 on the $j$-th element, as this amounts to scaling the amplitude of the input. If each control input acts on a single state, positively or negatively, the matrix $B$ will have the form

$$
\begin{aligned}
& B=\left[\begin{array}{lll}
B^{+} & -B^{-}
\end{array}\right], \text {where } \\
& B^{+}=\left[\begin{array}{llll}
b_{p_{1}} & b_{p_{2}} & \ldots & b_{p_{m}+}
\end{array}\right], \text { and } \\
& B^{-}=\left[\begin{array}{llll}
b_{q_{1}} & b_{q_{2}} & \ldots & b_{q_{m^{-}}}
\end{array}\right] .
\end{aligned}
$$

Define the index sets $\mathcal{P}=\left\{p_{1}, \ldots, p_{m^{+}}\right\}$and $\mathcal{Q}=$ $\left\{q_{1}, \ldots, q_{m^{-}}\right\}$, and consider the matrix $V$ as the combination of all real eigenvectors,

$$
V=\left[\begin{array}{lll}
V_{1} & \ldots & V_{l}
\end{array}\right],
$$

with $V_{i}, i=1, \ldots, l$, as in (7). With the given form of $B$, we have

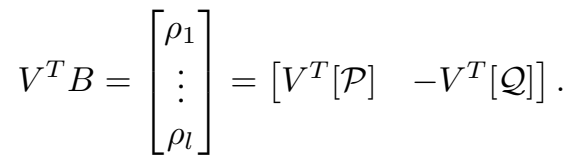

\section{B. Greedy Input Selection with Simple Real Eigenvectors}

When all real eigenvalues are simple, then $V_{i}=v_{i_{1}}, i=$ $1, \ldots, l$, and the rows of $V^{T} B$ are the row vectors $\rho_{i} \in$ $\mathbb{R}^{1 \times\left(m^{+}+m^{-}\right)}$. Notice that the input column $b_{j}, j \in \mathcal{P}$ (resp. $-b_{r}, r \in \mathcal{Q}$ ) corresponds to the column $V^{T}[j]$ (resp. $\left.-V^{T}[r]\right)$ in (14).

From Proposition 1, a procedure to construct $B$ must make sure that all rows of $V^{T} B$ are covered with a positive and a negative entry. Furthermore, we seek a minimum number of control inputs that accomplishes that.

This problem can be formulated as a variant of the well known combinatorial Set-Cover Problem [17]. This is an NP-complete problem, meaning that finding the exact global minimum becomes unfeasible when the size of the system grows. Greedy heuristics can be used instead. Such methods do not guarantee optimality, but in general result in a good approximation. A greedy algorithm is proposed for network control input selection also in [18].

Assume an initial control input matrix $B$ and the corresponding sets $\mathcal{P}$ and $\mathcal{Q}$ are given (possibly empty). Then $B$ is extended by adding input columns one at a time, and $\mathcal{P}$ and $\mathcal{Q}$ are updated accordingly. At every step of the iteration, the control input with the highest gain $\gamma^{*}=\max \left\{\gamma_{j}^{+}, \gamma_{r}^{-}\right\}$ is selected, where the gain $\gamma_{j}^{+}$(resp. $\gamma_{r}^{-}$) evaluates the contribution of the new positive control input candidate $b_{j}$ (resp. negative control input candidate $-b_{r}$ ). In formulas:

$$
\begin{aligned}
& \gamma_{j}^{+}=\kappa\left(\left[\begin{array}{ll}
V^{T}[\mathcal{P} \cup j] & \left.\left.-V^{T}[\mathcal{Q}]\right]\right)
\end{array}\right.\right.
\end{aligned}
$$

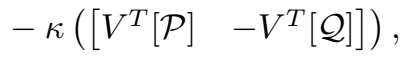

where $\kappa(\cdot)$ is the sum of the number of rows with a positive entry and the number of rows with a negative entry. $\gamma_{j}^{-}$is defined accordingly for a negative control input.

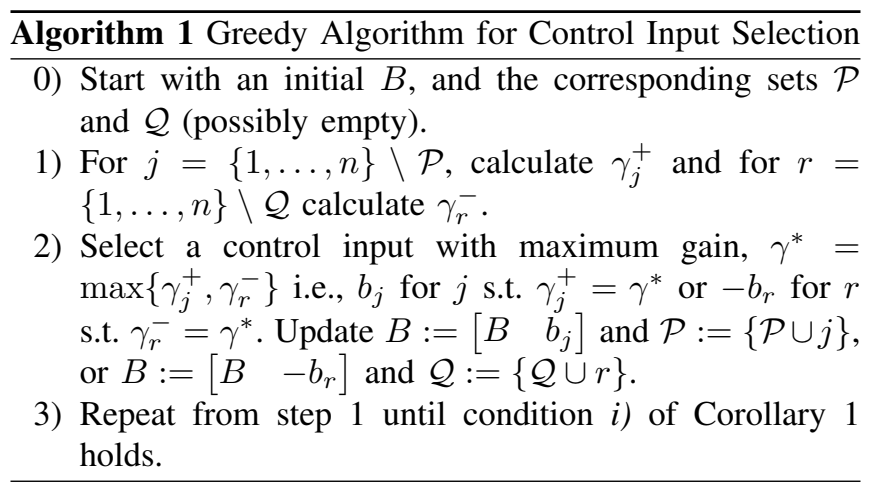

The procedure always terminate successfully in at most $2 l$ iterations.

Example: Assume $A^{T}$ has three real eigenvectors, grouped in the matrix

$$
V^{T}=\left[\begin{array}{ccc}
1 & 0 & 0 \\
1 & 1 & 0 \\
0 & 1 & -1
\end{array}\right] .
$$

After three iterations of Algorithm 1, we have

$$
\begin{aligned}
& B=\left[\begin{array}{lll}
b_{1} & b_{2} & -b_{1}
\end{array}\right], \\
& \mathcal{P}=\{1,2\} \text { and } \mathcal{Q}=\{1\} .
\end{aligned}
$$


In the fourth iteration of Algorithm 1, at step 1, we should calculate $\gamma_{3}^{+}, \gamma_{2}^{-}$and $\gamma_{3}^{-}$:

$$
\gamma_{3}^{+}=\kappa\left(\left[\begin{array}{cccc}
1 & 0 & 0 & -1 \\
1 & 1 & 0 & -1 \\
0 & 1 & -1 & 0
\end{array}\right]\right)-\kappa\left(\left[\begin{array}{ccc}
1 & 0 & -1 \\
1 & 1 & -1 \\
0 & 1 & 0
\end{array}\right]\right)=1 .
$$

In the same manner, $\gamma_{2}^{-}=1$ and $\gamma_{3}^{-}=0$. Therefore we can select the input column $b_{3}$ and extend $B$ to $\left[\begin{array}{ll}B & b_{3}\end{array}\right]$ and $\mathcal{P}$ to $\{\mathcal{P} \cup 3\}$ (step 2 in Algorithm 1). With this extension, we have

$$
\left[\begin{array}{l}
\rho_{1} \\
\rho_{2} \\
\rho_{3}
\end{array}\right]=\left[\begin{array}{cccc}
1 & 0 & 0 & -1 \\
1 & 1 & 0 & -1 \\
0 & 1 & -1 & 0
\end{array}\right]
$$

The condition $i$ ) of Corollary 1 is met and Algorithm 1 has finished.

\section{Extension for Multidimensional Real Eigenspaces}

From condition $i$ ) of Corollary 1, positive controllability requires that the columns of $\rho_{i}, i=1, \ldots, l$, positively span $\mathbb{R}^{\mu_{i}}$. This can be accomplished by the iterative construction of $B$ in Algorithm 1. However, the gain must be defined differently when $\rho_{i}$ is s.t. $\mu_{i}>1$. From equation (13) and (14), extending $B$ to $\left[\begin{array}{ll}B & b_{j}\end{array}\right]$ implies $\rho_{i}:=\left[\begin{array}{ll}\rho_{i} & \left.V_{i}^{T}[j]\right], i=\end{array}\right.$ $1, \ldots, l$.

Consider a step in our iterative construction process. $B$ consist of the columns of the already selected inputs. $\mathcal{P}$ and $\mathcal{Q}$ are computed accordingly, and $\rho_{i}, i=1, \ldots, l$, is calculated. Next, the contribution of extending $B$ to $\left[\begin{array}{ll}B & b_{j}\end{array}\right]$ must be evaluated. If the convex cone $\mathcal{C}\left(\rho_{i}\right) \subset \mathcal{C}\left(\left[\begin{array}{ll}\rho_{i} & V_{i}^{T}[j]\end{array}\right]\right)$ for any $i$, then including $b_{j}$ will bring us closer to meet condition $i$ ) of Corollary 1 .

In case there are several control inputs that enlarges $C\left(\rho_{i}\right)$ if included, a simple greedy selection strategy is used. Based on $m$ already selected control inputs, calculate the average direction of the vectors that positively $\operatorname{span} \mathcal{C}\left(\rho_{i}\right)$,

$$
\bar{\rho}_{i}=\frac{\sum_{r=1}^{m} \rho_{i}[r] /\left|\rho_{i}[r]\right|}{m}, i=1 \ldots l .
$$

Then calculate the gain of extending $B$ with $b_{j}$ as

$$
\hat{\gamma}_{j}^{+}=\sum_{i=1}^{l}\left(1-\left\langle\bar{\rho}_{i}, V_{i}^{T}[j]\right\rangle\right) .
$$

In (17), the inner product is a measure of distance between $V_{i}^{T}[j]$ and the central direction of $\mathcal{C}\left(\rho_{i}\right) . B$ is extended with the control input that maximizes this distance, all cones considered. Control inputs that are acting negatively on a node are of course evaluated correspondingly. After each extension of $\rho_{i}$ we must evaluate whether $C\left(\rho_{i}\right)$ positively span $\mathbb{R}^{\mu_{i}}$. When the gain is calculated in this way, Algorithm 1 can be applied to multidimensional eigenspaces. Algorithm 1 will always terminate successfully.

\section{Positive controllability: the Complete Algorithm}

Algorithm 1 provides a method to select inputs such that condition $i$ ) of Corollary 1 is met. When it comes to the rank condition, ii) of Corollary 1, the DM-decomposition and the analysis of the strongly connected components can be used for input selection.

Algorithm 2 states on a high level how Algorithm 1 can be combined with the DM-decomposition and with the analysis of the strongly connected components, in order to select inputs that make the system positively controllable. The graph $\mathcal{G}_{\Delta}$ considered here corresponds to the pair $\mathcal{U}$ and $\mathcal{E}$ defined in (4) and (5), constructed treating the current $A$ and $B$ as "generic" matrices. Similar considerations hold for the generic rank.

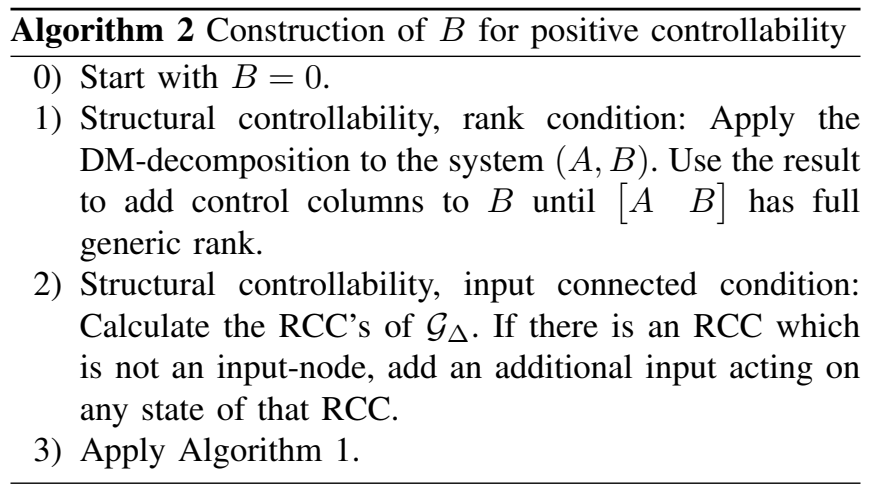

See e.g. [7] for a more detailed description on how step 1 and 2 are done. Note that the system $(A, B)$ may not be controllable although the corresponding structured system $\left(A_{\Delta}, B_{\Delta}\right)$ is structurally controllable. In such cases (a zeromeasure set) our Algorithm 2 will fail. It is always possible to replace step 1 and 2 with an exact construction method as in [6], at the cost of extra computational burden.

\section{Simulation Results}

In this section we apply Algorithm 2 to random networks and study how many positive control inputs are required for positive controllability. Denote $N_{d}^{r}$ such number. For comparison, we also compute the minimal number of driver nodes that are required for controllability, $N_{d}^{u}$, when $u$ is unrestricted.

We restrict our studies to weighted Erdős-Rényi networks with different edge-probabilities, $p$, and number of nodes, $n$. The edge weights are sampled from a normal distribution. In terms of the system matrix $A, A[i, j] \in N(0,1)$ with probability $p$, and $A[i, j]=0$ with probability $1-p$.

First, we study the case in which a network topology is given but the edge weights are sampled randomly. We want to see how much $N_{d}^{r}$ varies between networks with the same topology but different edge weights. The results are summarized in Table I. There are clearly variations in $N_{d}^{r}$. This is in contrast to $N_{d}^{u}$ that is always the same for a given topology as the theory of structural controllability suggests.

Next, we study how $N_{d}^{r}$ relates to network size. Both $N_{d}^{r}$ and $N_{d}^{u}$ decay with higher edge-probability, see Figure 1. This observation is well in line with the conclusions of [2], that $N_{d}^{u}$ is determined mainly by the degree distribution of the network.

Given the graph $\mathcal{G}(A)$ of a network, denote $N^{\mathrm{RCC}}$ the number of RCC's. As defined in Section II-C, $d_{r}$ is the rank 


\begin{tabular}{l|rrrrrr} 
Network Topology nr. & 1 & 2 & 3 & 4 & 5 & 6 \\
\hline Edge probability & 0.2 & 0.2 & 0.02 & 0.02 & 0.002 & 0.002 \\
No. nodes & 10 & 10 & 100 & 100 & 1000 & 1000 \\
\hline mean $\left(N_{d}^{r}\right)$ & 5 & 6 & 35.3 & 40.3 & 409.2 & 372.3 \\
$\operatorname{STD}\left(N_{d}^{r}\right)$ & 0 & 0 & 0.5 & 0.5 & 3.7 & 3.3 \\
\hline$N_{d}^{u}$ & 3 & 3 & 20 & 22 & 220 & 199 \\
& TABLE I
\end{tabular}

FOR SIX DIFFERENT ERDŐS-RÉNYI NETWORK TOPOLOGIES, 100 NETWORKS ARE GENERATED WITH RANDOM EDGE WEIGHTS.

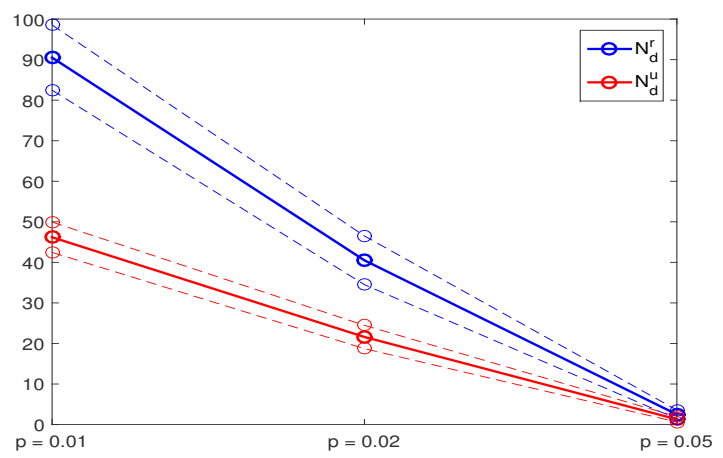

Fig. 1. $\quad N_{d}^{r}$ and $N_{d}^{u}$ for different edge probabilities, $p$. For every configuration, 100 random weighted Erdős-Rényi networks with 100 nodes are sampled. Mean and STD are calculated. Solid lines are mean-values, and dashed lines are mean \pm STD.

deficiency of $A$. Both $N^{\mathrm{RCC}}$ and $d_{r}$ are topological properties of the network. The sum $N^{\mathrm{RCC}}+d_{r}$ is an upper limit to the minimal number of unconstrained control inputs required for structural controllability [7]. Since any unconstrained control input is equivalent to one positive and one negative control input acting on the same node, $2\left(N^{\mathrm{RCC}}+d_{r}\right)$ control inputs is an upper bound in the case of positive controllability of a structured system. Simulations suggest that $N^{\mathrm{RCC}}+d_{r}$ is strongly related to $N_{d}^{r}$, see Figure 2 .

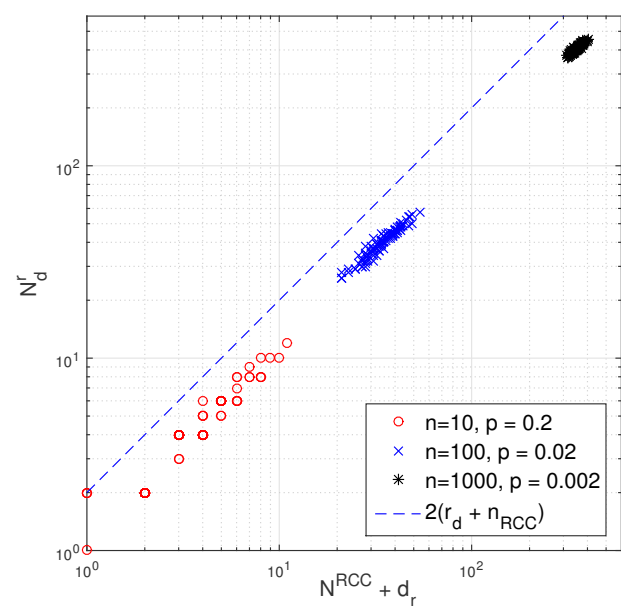

Fig. 2. Three network configurations are investigated. For each configuration 100 networks are generated and Algorithm 2 is applied. $N_{d}^{r}$ is compared with $N^{R C C}+d_{r}$.

\section{CONClusions}

Given an adjacency matrix $A$, we have developed an algorithm that successfully constructs an input matrix $B$ such that the resulting system $(A, B)$ is positively controllable with a near minimal number of positive control inputs. When the algorithm is applied to random weighted Erdős-Rényi networks, some interesting results are observed. The number of control inputs required for positive controllability is highly dependent on the network topology. But also the the edge weights matter, which is in contrast to when unconstrained control inputs are used. In the proposed algorithm, we have assumed that we are free to add positive or negative control inputs acting on any state. A related problem is to choose control inputs from a set of candidates. This is studied for structured systems in [19]. The proposed algorithm can be applied to such a problem with only minor adjustments.

\section{REFERENCES}

[1] R. E. Kalman, "Mathematical description of linear dynamical systems," Journal of the Society for Industrial \& Applied Mathematics, Series A: Control, vol. 1, no. 2, pp. 152-192, 1963.

[2] Y.-Y. Liu, J.-J. Slotine, and A.-L. Barabási, "Controllability of complex networks," Nature, vol. 473, no. 7346, pp. 167-173, 2011.

[3] A. Olshevsky, "Minimal controllability problems," Control of Network Systems, IEEE Transactions on, vol. 1, no. 3, pp. 249-258, Sept 2014.

[4] C. T. Lin, "Structural controllability," Automatic Control, IEEE Transactions on, vol. 19, no. 3, pp. 201-208, 1974.

[5] J.-M. Dion, C. Commault, and J. Van Der Woude, "Generic properties and control of linear structured systems: a survey," Automatica, vol. 39 , no. 7, pp. 1125-1144, 2003.

[6] Z. Yuan, C. Zhao, Z. Di, W.-X. Wang, and Y.-C. Lai, "Exact controllability of complex networks," Nat Commun, vol. 4, 092013.

[7] C. Commault and J.-M. Dion, "Input addition and leader selection for the controllability of graph-based systems," Automatica, vol. 49, no. 11 , pp. $3322-3328,2013$.

[8] M. Egerstedt, S. Martini, M. Cao, K. Camlibel, and A. Bicchi, "Interacting with networks: How does structure relate to controllability in single-leader, consensus networks?" Control Systems, IEEE, vol. 32, no. 4, pp. 66-73, Aug 2012.

[9] G. Notarstefano and G. Parlangeli, "Controllability and observability of grid graphs via reduction and symmetries," Automatic Control, IEEE Transactions on, vol. 58, no. 7, pp. 1719-1731, July 2013.

[10] D. Jacobson, Extensions of Linear-Quadratic Control, Optimization and Matrix Theory, ser. Mathematics in Science and Engineering. London: Academic Press, 1977, vol. 133.

[11] R. F. Brammer, "Controllability in linear autonomous systems with positive controllers," SIAM Journal on Control, vol. 10, no. 2, pp. 339-353, 1972

[12] M. Heymann and R. J. Stern, "Controllability of linear systems with positive controls: Geometric considerations," Journal of Mathematical Analysis and Applications, vol. 52, no. 1, pp. 36-41, 1975.

[13] Y. Ohta, H. Maeda, and S. Kodama, "Reachability, observability, and realizability of continuous-time positive systems," SIAM Journal on Control and Optimization, vol. 22, no. 2, pp. 171-180, 1984.

[14] C. Davis, "Theory of positive linear dependence," American Journal of Mathematics, vol. 76, no. 4, pp. pp. 733-746, 1954.

[15] K. Murota, "Systems analysis by graphs and matroids," in Algorithms and Combinatorics. Springer Verlag Berlin, 1987, vol. 3.

[16] S. E. Wright, "A note on positively spanning sets," The American Mathematical Monthly, vol. 107, no. 4, pp. 364-366, 2000.

[17] N. Alon, D. Moshkovitz, and S. Safra, "Algorithmic construction of sets for k-restrictions," ACM Transactions on Algorithms (TALG), vol. 2, no. 2, pp. 153-177, 2006.

[18] J. Gao, Y.-Y. Liu, R. M. D’Souza, and A.-L. Barabási, “Target control of complex networks," Nature communications, vol. 5, 2014.

[19] S. Pequito, S. Kar, and A. P. Aguiar, "On the complexity of the constrained input selection problem for structural linear systems," Automatica, vol. 62, pp. 193-199, 2015. 